MOUSQUER, C.J. et al. Desempenho reprodutivo de matrizes Nelore. PUBVET, Londrina, V. 8, N. 3, Ed. 252, Art. 1666, Fevereiro, 2014.

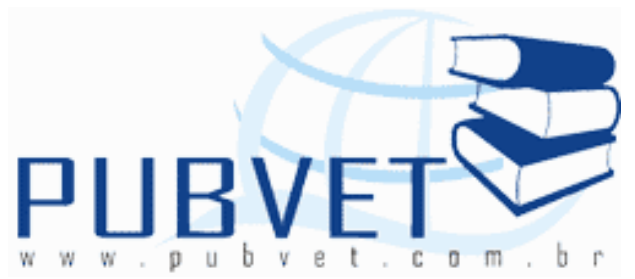

PUBVET, Publicações em Medicina Veterinária e Zootecnia.

\title{
Desempenho reprodutivo de matrizes Nelore
}

Claudio Jonasson Mousquer ${ }^{1 *}$, Fabíola Francisca Dias Fernandes², Geferson Antonio Fernandes ${ }^{3}$, Wanderson José Rodrigues de Castro ${ }^{1}$, Alvair Hoffmann ${ }^{4}$, Tiago Adriano Simioni ${ }^{4}$, Andrew Jonasson Mousquer ${ }^{5}$, Ronei Crispin Pequeno Gomes ${ }^{5}$

1 Mestrando em Ciência Animal da Universidade Federal de Mato Grosso, campus de Cuiabá-MT, Brasil

${ }^{2}$ Medica Veterinária - Autônoma, Sinop-MT, Brasil

${ }^{3}$ Zootecnista, Universidade Federal de Mato Grosso, campus de Sinop-MT, Brasil

${ }^{4}$ Mestrandos em Zootecnia da Universidade Federal de Mato Grosso, campus de Sinop-MT, Brasil

${ }^{5}$ Aluno de graduação em Medicina Veterinária, UFMT, Sinop-MT, Brasil

* correspondência: cjm@zootecnista.com.br

\section{Resumo}

O desempenho reprodutivo de vacas de cria é, de extrema importância para - aumento da produtividade da bovinocultura de corte, pois bezerros desmamados são um dos principais produtos comercializados pelos produtores em várias regiões do país. $\mathrm{O}$ desempenho reprodutivo das vacas de cria é influenciado por diversos fatores, porém, pode-se destacar como sendo um dos principais o nutricional, pelo balanço de energia que reflete na reserva de gordura corporal. Um programa de manejo nutricional adequado 
MOUSQUER, C.J. et al. Desempenho reprodutivo de matrizes Nelore. PUBVET, Londrina, V. 8, N. 3, Ed. 252, Art. 1666, Fevereiro, 2014.

deve proporcionar condição corporal desejável nos diferentes estágios de produção, cujas exigências aumentam significativamente no terço final da gestação e no intervalo de parição (início da lactação) até a reconcepção (pico da lactação). Contudo, objetivou-se avaliar as principais características reprodutivas de bovinos e destacar principais entraves da reprodução de matrizes, buscando primariamente possíveis soluções.

Palavras-chave: Nutrição, Reprodução, Estação de monta, Zootecnia

\title{
Reproductive performance of Nelore matrices
}

\begin{abstract}
The beef cows' reproductive performance has a big importance to increase the beef cattle productivity, because weaned calves are one of the leading products marketed by the producers in many regions of the country. The beef cows' reproductive performance is influenced by several factors however, the nutritional can be highlighted as one of the principal, because of the energy balance that reflects on fat body reserves. An adequate nutritional management program must provide a desirable body condition on different stages of the production, whose the requirements increase significantly in the final third of gestation and the farrowing interval (onset lactation) until the reconception (lactation peak). However, it was aimed to assess the main reproductive traits of cattle and highlight major barriers reproduction matrix, primarily seeking possible solutions.
\end{abstract}

Keywords: Nutrition, Reproduction, breeding season, Animal science

\section{Introdução}

O Brasil, devido às suas condições de clima, solo, topografia e, por suas tradições de séculos, tem no agronegócio uma de suas principais atividades econômicas que contribui efetivamente para seu desenvolvimento. A pecuária é um dos principais subsetores que compõe o produto interno bruto do país (PIB) e dentro dela a bovinocultura tem 
MOUSQUER, C.J. et al. Desempenho reprodutivo de matrizes Nelore. PUBVET, Londrina, V. 8, N. 3, Ed. 252, Art. 1666, Fevereiro, 2014.

importância fundamental, pois é composta por um dos maiores rebanhos do mundo e principal exportador de carne bovina.

O rebanho bovino brasileiro é constituído por, aproximadamente, $80 \%$ de animais de origem indiana e seus mestiços (Silva et al., 2002). A sua posição no contexto da pecuária mundial é a de segundo colocado em termos quantitativos, porém qualitativamente o seu desempenho apresenta baixos índices reprodutivos (60\% de taxa de nascimentos) e, em consequência, baixa produtividade (Fonseca, 2009).

O melhor desempenho reprodutivo depende de animais que iniciam a vida reprodutiva e que tenham o primeiro parto a uma idade mais precoce, dentro dos limites fisiológicos que não comprometam seu desempenho futuro, e que reiniciem a atividade ovariana num curto período pós-parto (Borges et al., 2009). Assim, pode-se obter maior produção de leite, carne e de crias durante a vida produtiva do animal.

Algumas vantagens são apontadas na literatura, em iniciar mais cedo à vida reprodutiva das novilhas. Desta forma uma menor idade ao primeiro parto, atua de forma direta e expressiva na taxa de desfrute, estando assim, correlacionada à eficiência e lucratividade da produção, além de possibilitar maior intensidade de seleção, menor intervalo de gerações, longevidade da fêmea no rebanho e número de fêmeas para reposição (Mousquer et al.,2013).

Os prejuízos mais visíveis e fáceis de serem identificados na pecuária de corte ocorrem quando as fêmeas não conseguem emprenhar, ou quando há perdas no período próximo ou imediato ao parto. Entretanto, outros fatores de igual importância devem ser considerados, tais como antecipação dos partos na eficiência reprodutiva da estação de monta subsequente, valorização e ganho genético das novilhas de reposição, aumento do peso ao desmame dos bezerros, diminuição da idade ao abate de novilhos (TorresJunior et al., 2009).

A grande maioria dos produtores desconhece a importância e a maneira de se efetuar um efetivo desempenho reprodutivo de suas matrizes, 
MOUSQUER, C.J. et al. Desempenho reprodutivo de matrizes Nelore. PUBVET, Londrina, V. 8, N. 3, Ed. 252, Art. 1666, Fevereiro, 2014.

bem como várias técnicas de manejo e de cuidados com a alimentação, disponíveis e indispensáveis à melhoria da eficiência na atividade da pecuária. Cabe aos técnicos (Zootecnistas, Veterinários, Agrônomos, dentre outros) a grande responsabilidade de reverter a situação atual, levando ao conhecimento dos produtores modernas técnicas ou informações capazes de melhorar os índices zootécnicos do rebanho.

\section{Revisão Bibliográfica}

O crescimento rápido é uma característica desejável na bovinocultura de corte, pois os animais que têm maior capacidade de crescimento precisam de menor período de tempo para atingir a puberdade e assim a idade de abate.

Sendo assim, há uma necessidade crescente de melhorar substancialmente a produtividade do rebanho nacional, principalmente no que diz respeito ao desempenho reprodutivo, que é de fundamental importância na taxa de desfrute e na determinação de maior ou menor disponibilidade de animais para seleção (Szechy et al., 1995).

As características reprodutivas são em sua grande maioria, difíceis de serem mensuradas e interpretadas, por em muitas razões, serem influenciadas por diferentes fatores de interação entre o bezerro, vaca, touro (Mercadante et al., 2000) e também o ambiente. Contudo, podemos destacar que, a eficiência reprodutiva dos rebanhos é um dos fatores

determinantes na produção e deve, portanto, ser considerada até mesmo como critério de seleção em programas de melhoramento animal.

Nos trópicos, a idade ao primeiro parto (IPP) tardia é um dos principais fatores que afetam negativamente a produção de carne (Azêvedo et al., 2006). Com isso destaca-se que a IPP em bovinos de corte é uma variável que afeta a produtividade dos sistemas de cria, uma vez que reduz o número de novilhas em recria (Potter et al., 1998) e em consequência disso, aumenta a produtividade da vaca. 
MOUSQUER, C.J. et al. Desempenho reprodutivo de matrizes Nelore. PUBVET, Londrina, V. 8, N. 3, Ed. 252, Art. 1666, Fevereiro, 2014.

Durante algum tempo a maioria dos programas de melhoramento genético de bovinos, focou sobre as características de crescimento do animal. Apesar de também ser de grande importância econômica, as características reprodutivas foram pouco utilizadas nestes programas, em consequência da sua baixa herdabilidade e da dificuldade de mensuração, que se deve ao fato de haver forte influência do meio ambiente.

A reprodução animal junto ao melhoramento genético, permanecem no topo da pirâmide da produção animal, logo definem a qualidade dos animais utilizados (Britt, 1986) e, estratégias que irão influenciar todo sistema de produção.

Alem do melhoramento genético, biotécnicas de reprodução surgem com o intuito de alavancar todo sistema de produção na pecuária. A inseminação artificial mostrar-se como um importante instrumento para a melhoria do patrimônio genético, e para implementação dos índices produtivos dos rebanhos de bovinos de corte, em propriedades com elevado número de animais a ser inseminados, como observado em grande parte das propriedades do Centro-oeste brasileiro, alguns problemas com a detecção de cio, ou mesmo com a mão de obra podem limitar esta técnica, visto que apenas $11 \%$ dos rebanhos nos países do Mercosul são inseminados (Nogueira et al., 2011).

Alem da inseminação, outras biotécnicas vem sendo cada vez mais empregadas em vários rebanhos no Brasil e, em grandes rebanhos no centro oeste brasileiros como a transferência de embriões (TE) através da técnica de superovulação (SOV) e a Fertilização in vitro (FIV) são também ferramentas que possibilitam aumentar o potencial reprodutivo de fêmeas consideradas de genética superior (Nogueira et al., 2011), principalmente como instrumento de manejo para acelerar o processo de melhoramento genético e de programas de seleção animal.

Sem dúvidas é fato destacar o aumento dos custos de produção no setor da pecuária, influenciados pela ocupação de áreas de pastagens pela agricultura de grãos (Vieira et al., 2005), em consequência ao aumento da 
MOUSQUER, C.J. et al. Desempenho reprodutivo de matrizes Nelore. PUBVET, Londrina, V. 8, N. 3, Ed. 252, Art. 1666, Fevereiro, 2014.

população também há o aumento crescente nas vendas de carne bovina ao exterior e o potencial do mercado interno determinam ao produtor a conscientização da necessidade de maior produção e produtividade nos rebanhos de cria, sofrendo todo o impacto as categorias subsequentes de recria e engorda, acarretando na produção de matrizes.

\section{Definições de Estratégias para o Desempenho Reprodutivo}

Um dos maiores desafios entre os produtores é melhorar a eficiência reprodutiva das matrizes de corte, a qual pode ser obtida por meio de seleção para reduzir a idade ao primeiro parto. Entre as principais vantagens em emprenhar novilhas mais jovens, destacam-se o aumento da vida reprodutiva da vaca e do número de bezerros e a redução do tempo para obter retorno do investimento (Short et al., 1994).

Os aumentos da produtividade na pecuária de corte passam, obrigatoriamente, pelas correções dos diversos manejos, como um bom manejo profilático e um bom manejo alimentar, projetado de maneira a atender as necessidades nutricionais dos animais, com menor custo e de modo adaptado à realidade local de produção. Além disso, um bom manejo reprodutivo é necessário para que os animais expressem todo o seu potencial produtivo, de forma ritmada e contínua (Brito et al., 2009).

A seleção e o manejo de novilhas e vacas para reprodução contemplam uma série de decisões que devem estar relacionadas as características particulares de cada animal, como, por exemplo, idade e peso corporal, época de parição, escore de condição corporal (a fêmea não deve estar nem muito magra nem muito gorda) e condição uterina/ovariana (avaliada por meio do exame ginecológico). Esses fatores serão determinantes da fertilidade geral do rebanho e das chances de as fêmeas emprenharem, pois influenciam diretamente a puberdade em novilhas e o anestro pós-parto nas vacas (período em que a vaca permanece sem apresentar cio). Além disso, a seleção mais acurada de fêmeas passa pela 
MOUSQUER, C.J. et al. Desempenho reprodutivo de matrizes Nelore. PUBVET, Londrina, V. 8, N. 3, Ed. 252, Art. 1666, Fevereiro, 2014.

precocidade sexual (idade ao primeiro cio), habilidade materna (desmamar bezerros pesados) e capacidade de produzir um bezerro por ano.

Um baixo percentual de vacas com cria acarretará baixo retorno financeiro do empreendimento, requerendo-se assim a adoção de medidas capazes de corrigir as distorções que causam essa situação visando a estabilização do rebanho.

Uma característica que torna importante para o sistema pecuário e, obter bons índices zootécnicos e possíveis correções no desempenho reprodutivos das matrizes são as anotações (Tabela 1), onde um mínimo necessário de informações se torna muito importante para futuras correções nos índices zootécnicos e estratégias para o bom desempenho.

Tabela 1. Anotações mínimas necessárias para um bom controle do desempenho reprodutivo.

Ocorrência

parto

estro (cio)

cobrição ou inseminação

artificial

abortos

outras ocorrências

tratamentos
O que anotar

Nome e número da vaca, data, condição do

$$
\text { parto, sexo da cria }
$$

Data, nome e número da vaca

Nome e número da vaca, data, nome e número do touro ou identificação do

Sêmen

Data, nome e número da vaca Infecções uterinas, cistos, retenção de placenta...

Nome e número da vaca, data e tratamentos efetuados 
MOUSQUER, C.J. et al. Desempenho reprodutivo de matrizes Nelore. PUBVET, Londrina, V. 8, N. 3, Ed. 252, Art. 1666, Fevereiro, 2014.

\section{Puberdade}

O crescimento rápido é uma característica destacável na bovinocultura de corte, pois os animais que têm maior capacidade de crescimento precisam de menor período de tempo para atingir a puberdade (reproduzindo-se antecipadamente) e também a idade ao abate, com melhores acabamento de carcaça. Dessa forma, os ganhos médios diários devem ser estudados e incluídos em programas de seleção (Sarmento et al., 2003), e enfatizados as futuras reprodutoras do plantel .

A puberdade na fêmea é definida como o início da ciclicidade reprodutiva. Ela é consequência de uma série acumulativa de eventos hormonais e está mais associada com o peso corporal que com a idade. Para a puberdade é o tempo que ocorre o primeiro estro acompanhado de ovulação. A idade do primeiro cio em novilhas varia consideravelmente, sendo dependente da raça, da nutrição e da taxa de crescimento. Um baixo nível nutricional e de crescimento adia o aparecimento da puberdade, assim como alto nível nutricional e rápido crescimento acelera o seu aparecimento. A idade média para o aparecimento da puberdade é quando atingirem cerca de 30 a $40 \%$ do peso adulto gerando em torno de 10 a 12 meses para raças leiteiras e entre 11 a 15 para raças de corte. Vale ressaltar que essas fêmeas só estarão aptas para reprodução seja por monta natural ou inseminação artificial quando atingirem cerca de 70 \% do peso vivo de um animal adulto.

\section{Escore da Condição Corporal (ECC)}

O escore da condição corporal é um método subjetivo, de fácil avaliação do estado nutricional dos animais, ao qual se atribuem valores numéricos. A condição corporal é obtida por observação e palpação da gordura subcutânea das costelas, processos espinhosos e transversos das vértebras lombares e/ou dorsais, tuberosidade isquiática e sacral e inserção de cauda. 
MOUSQUER, C.J. et al. Desempenho reprodutivo de matrizes Nelore. PUBVET, Londrina, V. 8, N. 3, Ed. 252, Art. 1666, Fevereiro, 2014.

Santos et al. (2009) descreve que um programa de manejo nutricional adequado deve proporcionar condição corporal desejável nos diferentes estágios de produção, cujas exigências aumentam significativamente no terço final da gestação e no intervalo de parição (início da lactação) até a reconcepção (pico da lactação). A avaliação do escore de condição corporal permite a análise das práticas de manejo adotadas e pode fornecer subsídios aos produtores na melhoria e na eficiência dos programas de manejo reprodutivo e nutricional.

Como no período pré-parto (60-90 dias antes da parição) as exigências energéticas das vacas de corte ainda são baixas, esse é o período mais adequado para adotar estratégias de manejo nutricional, pois nesta fase as vacas podem armazenar gordura de forma mais rápida e econômica. Neste período, as matrizes devem ser separadas por meio do ECC, ou seja, as vacas com ECC baixo, devem ser colocadas em pastagens de melhor qualidade e/ou receber um plano de manejo nutricional adequado (Santos et al., 2009).

\section{Estação de Monta}

Nas condições tropicais do Brasil, o regime de chuvas é quem define os períodos mais marcantes para o setor agropecuário (período seco e período chuvoso). Dentro desta variação anual, a disponibilidade de forragem em quantidade e qualidade suficientes para alimentar os animais direciona a época mais adequada às atividades de monta, nascimentos e desmame. O déficit nutricional pode prejudicar substancialmente o desenvolvimento dos bezerros e a fertilidade das fêmeas, prolongando o intervalo de partos devido ao aumento do período de serviço (intervalo parto-concepção).

Nas condições do Brasil Central, o período de estação de monta se dá entre outubro e dezembro, e os nascimentos se concentram de julho a setembro (período seco). Desta forma, nas propriedades que preconizam o desmame aos oito meses de idade, os bezerros são separados das mães nos 
MOUSQUER, C.J. et al. Desempenho reprodutivo de matrizes Nelore. PUBVET, Londrina, V. 8, N. 3, Ed. 252, Art. 1666, Fevereiro, 2014.

meses de março, abril e maio, época de grande oferta de pastagens. Esta prática favorece o desempenho do bezerro no início da recria, bem como o da vaca, que estará sem bezerro ao pé e poderá suportar melhor as restrições do período seco e melhorar a condição corporal ao parto (Valle et al., 2000).

A duração da estação de monta também é importante para se verificar a precocidade e a fertilidade das fêmeas. Tal período não deve ultrapassar 90 dias para vacas (tabela 2) e 60 dias para novilhas, pois, nestes casos, as fêmeas terão chance suficiente de apresentar entre 2 e 4 cios férteis e emprenharem (duração média de 21 dias entre cios).

Tabela 2. Parâmetros reprodutivos para rebanhos de corte.

Parâmetro

Extensão da estação de monta

Taxa de prenhez ( 35 dias após o término da estação de monta)

\section{Porcentagem de bezerros nascidos vivos} (das vacas confirmadas gestantes)

\section{Objetivo}

$$
<90 \text { dias }
$$$$
>90 \%
$$$$
>93 \%
$$

\section{Período de Serviço (PS) e Intervalo entre Partos (IEP)}

O período de serviço, ou dias em aberto, é definido como período (em dias) entre o parto até a primeira concepção fértil confirmada pela gestação da vaca. O período de serviço é influenciado diretamente pela fertilidade da fêmea e do macho, pela eficiência de deteç̧ão de cio e pela inseminação artificial. Como o período de gestação nos bovinos não sofre grandes variações, em média 292 dias, o intervalo entre parto, é considerado o indicador final da performance reprodutiva de um rebanho, está diretamente 
MOUSQUER, C.J. et al. Desempenho reprodutivo de matrizes Nelore. PUBVET, Londrina, V. 8, N. 3, Ed. 252, Art. 1666, Fevereiro, 2014.

relacionado a produção, como pode ser visualizado na interpretação dos dias em aberto na tabela 3.

Tabela 3. Interpretação dos dias em aberto, para melhor interpretação dos índices zootécnicos.

Dias em Aberto

$<85$ dias

85 a 115 dias

116 a 130 dias

131 a 145 dias

\section{Interpretação}

Bom

Problemas pequenos para a maioria dos

rebanhos

Problemas moderados para a maioria dos

rebanhos

As vacas que mantêm ou perde condição corporal tem um prolongado intervalo entre parto e o primeiro estro pós-parto, são menos férteis e produzem menores bezerros a desmama.

Com o IEP ideal (12 meses), ter-se-ia a produção de um bezerro por vaca por ano, numero estabelecido pelo limite biológico em condições naturais de reprodução na espécie bovina, mas no sistema de bovinocultura de corte do Brasil, predomina o IEP mais longo que o ideal.

\section{Idade ao Primeiro Parto (IPP)}

A antecipação da idade ao primeiro parto está diretamente ligada à eficiência e à lucratividade da produção de carne bovina. Novilhas que parem mais cedo têm maior vida produtiva que as fêmeas mais tardias, assim conseqüentemente, novilhas que parem pela primeira vez aos dois anos de idade deverão produzir mais bezerros do que as que parem aos três anos de idade. 
MOUSQUER, C.J. et al. Desempenho reprodutivo de matrizes Nelore. PUBVET, Londrina, V. 8, N. 3, Ed. 252, Art. 1666, Fevereiro, 2014.

Selecionar Matrizes pela precocidade sexual das fêmeas não é simples, pois as características reprodutivas, geralmente, têm baixas herdabilidades e não são facilmente mensuradas. Além disso, alguns produtores atrasam a entrada das fêmeas na reprodução determinando uma idade ou um peso para que estas iniciem sua vida reprodutiva, dificultando ainda mais a identificação das fêmeas mais precoces sexualmente (Dias et al., 2004).

Segundo Dias et al. (2004) a idade ao primeiro parto é uma característica que pode ser utilizada como critério de seleção, por estar relacionada com a puberdade dos animais. Além disso, a obtenção desta característica não implica em custo para o sistema.

Vacas paridas tarde em um ano, tendem a parir mais tarde ou não parir no ano seguinte. Dessa forma as novilhas devem entrar em serviço antes das vacas com o objetivo de parir mais cedo e aumentar sua eficiência de produção. Esta pratica resulta, frequentemente, em novilhas concebendo nos primeiros ciclos estrais após a puberdade no sistema 13/15 meses de idade (Patterson et al., 1992).

As Novilhas devem ser alimentadas até atingir cerca de $70 \%$ do peso vivo de uma vaca adulta e condições corporais moderadas, para dar inicio a sua primeira estação de acasalamento.

A elevada idade ao primeiro parto (IPP) esta diretamente relacionado aos fatores ambientais e nutrição, sendo determinante para todo manejo reprodutivo subsequente. Quanto à nutrição, deve-se salientar que um dos principais motivos para o aparecimento tardio da puberdade nos rebanhos zebuínos no Brasil é a estacionalidade, caracterizado pela sazonalidade da produção de forragens, onde temos alta oferta de forragem no período das águas e muito baixa oferta de forragem na seca, associada à ausência de suplementação alimentar na primeira estação seca após o desmame, quando a fêmea ainda está em crescimento.

Segundo Azevedo et al. (2006) nos rebanhos avaliados, a idade média ao primeiro parto $(45,14$ meses $)$ denota que as novilhas estão, 
MOUSQUER, C.J. et al. Desempenho reprodutivo de matrizes Nelore. PUBVET, Londrina, V. 8, N. 3, Ed. 252, Art. 1666, Fevereiro, 2014.

provavelmente, sendo acasaladas pela primeira vez aproximadamente aos três anos de idade, o que é bastante tarde para o primeiro acasalamento.

Silva et al. (2005) avalia alternativa para reduzir a idade ao primeiro acasalamento dos 24 para os 14-15 meses de idade, pode-se utilizar sistemas intermediários com o primeiro serviço aos 18 meses de idade, em uma outra estação reprodutiva. Nesse sistema, os custos com a criação das novilhas são menores que os obtidos no sistema 14-15 meses. O mesmo autor comenta que é viável a antecipação da idade ao primeiro acasalamento de 24 para 18 meses de idade, porém para obtenção de melhores índices reprodutivos no acasalamento aos 18 meses, são necessárias melhorias na fase de recria das fêmeas bovinas, para que alcancem maior peso no primeiro acasalamento.

\section{Considerações Finais}

Mesmo obtendo resultados cada vez mais satisfatórios na pecuária brasileira, há necessidade de identificação de mais fatores determinantes da eficiência reprodutiva de matrizes que compõe o rebanho brasileiro, gerando informações que possam ser utilizadas por maior numero de produtores, contribuindo para tornar a pecuária uma atividade cada vez mais rentável.

Na reprodução animal, qualquer que seja a espécie ou raça explorada, é indispensável que todo o esforço seja dirigido para que sejam alcançados dois objetivos básicos: a elevada produtividade e também a produção de qualidade. Evidentemente, essas duas metas resultam em maior rentabilidade da exploração na pecuária de corte.

\section{Referências Bibliográficas}

AZEVEDO, D. M. M. R.; FILHO, R. M.; LOBO, R. N. B.; MALHADO, C. H. M.; LOBO, R. B.; MOURA, A. A. A.; FILHO, E. C. P. Desempenho Reprodutivo de Vacas Nelore no Norte e Nordeste do Brasil. Revista Brasileira de Zootecnia, v.35, n.3, p.988-996, 2006.

BENEDETTI, E.; SILVA, H. S. Influencia da condição corporal na produção de leite, consumo e desempenho reprodutivo de vacas leiteiras. Vet. Not. Uber., v.3, n.1, p.175-183, 1997. 
BOLIGON, A. A.; ALBUQUERQUE, L. G.; RORATO, P. N. R. Associações genéticas entre pesos e características reprodutivas em rebanhos da raça nelore. Revista Brasileira de Zootecnia., v.37, n.4, p.519-530, 2008.

BORGES, A. M.; CARVALHO, B. C.; RUAS, J. R. M. Manejo reprodutivo da vaca mestiça: estado da arte. Revista Brasileira de Reprodução Animal, n.6, p.157-162, 2009 (supl.)

BRITO, A. S.; NOBRE, F. V.; FONSECA, J. R. R. Bovinocultura Leiteria: Informações técnicas e de gestão. (Orgs.). - Natal: SEBRAE/RN, 2009. 320 p.

BRITT, J. H. Increasing efficiency through improved reproduction: current technology in reproductive management. Journal of Animal Science, Champaign, p. 34-45, 1986.

DIAS, L. T.; EL FARO, L.; ALBUQUERQUE, L. G. Estimativa de Herdabilidade para Idade ao Primeiro Parto de Novilhas da Raça Nelore. Revista Brasileira de Zootecnia, v.33, n.1, p.97-102, 2004.

FONSECA, V. O. Avaliação reprodutiva de touros para monta a campo: análise crítica. Revista Brasileira de Reprodução Animal, n.6, p. 36-41, 2009 (supl.)

MERCADANTE, M. E. Z.; LÔBO, R.; OLIVEIRA, H. N. Estimativas de (co)variâncias entre características de reprodução e de crescimento em fêmeas de um rebanho Nelore. Revista Brasileira de Zootecnia, v.29, n.4, p.997-1004, 2000.

MOUSQUER, C. J.; ARAUJO, C. V.; ARAUJO, S. I.; COTRIM, T. S.; LOBO, R. B.; BEZERRA, L. F. Idade ao primeiro parto e pesos ao ano e sobreano na raça Nelore. In: REUNIÃO ANUAL DA SOCIEDADE BRASILEIRA DE MELHORAMENTO ANIMAL, 10, 2013, Uberaba, Anais... Uberaba: SBMA, 2013. (CD-ROM)

NOGUEIRA, E.; DIAS, A. M.; ÍTALO, L. C. V. Biotécnicas aplicadas a reprodução animal. 2011, I Simpósio Sulmatogrossense de produção animal. VIII Semana da Zootecnia, Universidade Católica Dom Bosco - UCDB, 2011.

OLIVEIRA, L. D. Estudo da influencia de fatores genéticos e ambientais sobre as características produtivas e reprodutivas em um rebanho de bovinos de raça nelore no estado de Goiás. Brasília-DF: UnB, 2007. 56 p. (Dissertação de Mestrado).

PATTERSON, D. J., PERRY, R. C. KIRACOFE, G. H. et al. Management considerations in heifer development and puberty. Journal of Animal Science, v.70, n.12, 4018-4035, 1992.

POTTER, L.; LOBATO, J. F. P.; NETTO, C. G. M. Produtividade de um modelo de produção para novilhas de corte primíparas aos dois, três ou quatro anos de idade. Revista Brasileira de Zootecnia, v.27, n.3, p.613-619, 1998.

SANTOS, S. A.; ABREU, U. G. P.; SOUZA, G. S.; CATTO, J. B. Condições corporal, variação de peso e desempenho reprodutivo de vacas de cria em pastagem nativa no pantanal. Revista Brasileira de Zootecnia, v.38, n.2, p. 354-360, 2009.

SARMENTO, J. L. R.; PIMENTA FILHO, E. C.; RIBEIRO, M. N.; MARTINS FILHO, R. Efeitos Ambientais e Genéticos Sobre o Ganho em Peso Diário de Bovinos Nelore no Estado da Paraíba. Revista Brasileira de Zootecnia, v.32, n.2, p.325-330, 2003. 
SILVA, L. O. C.; GONDO, A.; NOBRE, P. R. C.; EUCLIDES FILHO, K.; ROSA, A. N.; JOSAHKIAN, L. A.; FIGUEIRED, G. R. Genetic Trends in Nelore Breed in Brazil. In: World Congress of Genetics Applied to Livestock Production, 7., 2002, Montpellier, França. Proceedings... Montpellier, 2002. p. 321-332.

SILVA, M. D.; BARCELLOS, J. O. J.; PRATES, E. R. Desempenho reprodutivo de novilhas de corte acasaladas aos 18 ou 24 meses de idade. Revista Brasileira de Zootecnia, v.34, n.6, p. 2057-2063, 2005.

SHORT, R. Y.; STAIMILLER, R. B.; BELLOWS, R. L. et al. Breeding heifers at one year of age: biological and economic considerations. In: FIELDS, M.J.; SAND, R.S. (Eds.) Factors affecting calf crop. London: CRC Press, 1994. p.55-68.

SZÉCHY, M. L. M., BENEVIDES FILHO, I. M., SOUZA, L. M. Idade ao primeiro parto, intervalo de partos e peso ao nascimento de um rebanho Nelore. Revista Brasileira de Ciências Veterinária, v.2, n.2, p.47-49, 1995.

TORRES-JUNIOR, J. R. S.; MELO, W. O.; ELIAS, A. K. S.; RODRIGUES, L. S.; PENTEADO, L.; BARUSELLI, P. S. Considerações técnicas e econômicas sobre reprodução assistida em gado de corte. Revista Brasileira de Reprodução Animal, v.33, n.1, p. 53-58, 2009.

VALLE, E. R,; ANDREOTTI, A. R.; THIAGO, R. L. S. Técnicas de manejo reprodutivo em bovinos de corte. Campo Grande, MS: Embrapa Gado de Corte, 2000. 61p.

VIEIRA, A.; LOBATO, J. F. P.; TORRES JUNIOR, R. A. A.; CEZAR, I. M.; CORREA, E. S. Fatores Determinantes do Desempenho Reprodutivo de Vacas Nelore na Região dos Cerrados do Brasil Central. Revista Brasileira de Zootecnia, v.34, n.6, p.2408-2416, 2005 (supl). 